Europhysics Letters

PREPRINT

\title{
Non-linear terms in 2D cosmology
}

\author{
F. P. Devecchi $\left(^{*}\right)$ and M. L. Froehlich \\ Departamento de Física, Universidade Federal do Paraná, Caixa Postal 19044, 81531-990, \\ Curitiba, Brazil[may 2005]
}

PACS. 04.50.th; 98.80. Elosmological models in lower dimensions.

\begin{abstract}
. -
In this work we investigate the behavior of two-dimensional (2D) cosmological models, starting with the Jackiw-Teitelboim (JT) theory of gravitation. A geometrical term, non-linear in the scalar curvature $R$, is added to the JT dynamics to test if it could play the role of dark energy in a 2D expanding universe. This formulation makes possible, first, the description of an early (inflationary) $2 \mathrm{D}$ universe, when the van der Waals ( $\mathrm{vdW}$ ) equation of state is used to construct the energymomentum tensor of the gravitational sources. Second, it is found that for later times the non-linear term in $R$ can generate an old $2 \mathrm{D}$ universe in accelerated expansion, where an ordinary matter dominated era evolves into a decelerated/accelerated transition, giving to the dark energy effects a geometrical origin. The results emerge through numerical analysis, following the evolution in time of the scale factor, its acceleration, and the energy densities of constituents.
\end{abstract}

Introduction. - The study of theories of gravity in lower dimensions has received extensive consideration in the literature [1]. Taking the particular case of two-dimensional (2D) theories, the gravity formulation proposed by Teitelboim and Jackiw [2] (JT model) provided consistent results at the classical and quantum levels [1]. This model is the most imediate substitute of general relativity in 2D field formulations, since the Einstein theory furnish no dynamics in 2D [1]. The JT model was under investigation also in a cosmological context $[3,6]$. The results obtained include the description of a Universe filled with ordinary matter or/and electromagnetic radiation, an inflationary 2D universe and a three-eras universe where a cosmological constant was responsible for a final accelerated period, that could de associated to the effects of dark energy [3].

In fact, the dark energy problem, as a fundamental discussion in cosmology, has been analised using different approaches. Recently, it was proposed a geometrical formulation for the dark energy behavior in a $4 \mathrm{D}$ universe [5]. This idea comes in substitution of the standard formulations that takes the dark energy as an usual source [5]. This geometrical formulation has as central point a modification in the Einstein action that includes a non-linear term in the scalar curvature $R$, that would be key ingredient behind the present accelerated expansion in the universe [5].

$\left(^{*}\right)$ devecchi@fisica.ufpr.br

(c) EDP Sciences 
The proposal of our work is to make an analogous modification in the JT model, and analyse in which cases the non-linear term in $R$ is responsible for a final period of positive acceleration. In those cases the geometrical term would be a substitute to the cosmological constant in playing the role of dark energy in 2D universes. Following our previous effort [6], we model the inflaton-matter source using the van der Waals equation of state ( $\mathrm{vdW}$ ), that is responsible for an initial strongly accelerated period (first period) corresponding to an inflationary young $2 \mathrm{D}$ universe (dominated by the inflaton). As the system evolves the vdW equation approaches a barotropic equation of state (modelling a period where matter/radiation start to predominate over the inflaton contribution), producing a decelerated expansion of the $2 \mathrm{D}$ universe (second period). In these two periods the non-linear term in $R$ gives no sensible contribution to the dynamics, and the standard JT results [6] are reproduced. It is in a final (third) period that the non-linear term promotes, in some situations, an accelerated era as a geometrical effect; so the usual negative-pressure sources are not needed to simulate the dark energy dominated period. In the following section we make a brief review of the JT model in cosmology that works as preface to the presentation of our results.

The Jackiw-Teitelboim model and cosmology. - As it was mentioned in the introduction the Jackiw-Teitelboim (JT) model [2] is a succesful formulation of 2D gravity. Among other results, that include a consistent quantum version, the model is able to furnish interesting results in $2 \mathrm{D}$ cosmologies $[3,6]$. The dynamics of the JT theory is ruled by

$$
R(x)=-\kappa T_{\mu}^{\mu}(x)+\Lambda,
$$

where $R$ is curvature scalar, $T^{\mu \nu}$ is the energy momentum tensor of the sources, and $\Lambda$ is a cosmological constant. The proposal of the JT model (in substitution to the Einstein field equations that in 2D are obsolete [1]) focused on the fact that the full geometrical information for the 2D space-time is encapsulated in the curvature scalar $R$ and as a consequence the gravitational field dynamics can be put in a scalar form. The above mentioned cosmological applications consider an hypothetical 2D Universe where the assumptions of spatial homogeneity and isotropy are invoked. The Robertson-Walker metric has the following form in a 2D Riemannian space-time

$$
d s^{2}=(d t)^{2}-a(t)^{2}(d x)^{2},
$$

where $a(t)$ is the cosmic scale factor, that encapsulates the complete information about the evolution of the gravitational field created by the sources. The equations of motion (together with the conservation law $T_{; \nu}^{\mu \nu}=0$ ) can be expressed as (making $\kappa=2 \pi G_{1}$ with $G_{1}=1$ )

$$
\frac{\ddot{a}}{a}=-4 \pi(\rho-p)+\frac{\Lambda}{2}, \quad \dot{\rho}+\frac{\dot{a}}{a}(\rho+p)=0,
$$

where $\rho$ and $p$ are the energy density and the hidrostatic pressure of the sources, respectively. The cosmological solutions of the JT model include an old universe filled with radiation and/or matter [3] (a 2D counterpart of the results found in [4]); inflationary scenarios (with transitions to a matter era) are also possible, when the cosmic fluid obeys the van der Waals (vdW) equation of state [6]. Another interesting result is that a three eras regime (a first strongly accelerated period followed by a decelerated one dominated by matter/radiation; and a final accelerated era) can be obtained when a cosmological constant $\Lambda$ is included. Here this constant is playing the role of a dark energy density that must always have negative values ( a situation that occurs only in $2 \mathrm{D}[6]$ ).

The $1 / R$ correction in $2 D$. - Our proposal is that The JT model dynamics be corrected by a term of the form $\omega^{2} / R$. This dynamics is inspired by the recent investigations [5] of giving a geometrical origin to the dark energy constituent in $4 \mathrm{D}$ cosmological models. Here the contribution of the non-linear term becomes important for later times, promoting an acelerated expansion period, making possible, at least qualitatively, the association with the dark energy contributions. In the 
$2 \mathrm{D}$ case we want to investigate the effects produced by the non-linear term in $R$ and in which cases this can be associated to a dark-energy contribution. The field equation read in this case

$$
R-\frac{\omega^{2}}{R}+\kappa T=0 \Longrightarrow R=\frac{-\kappa T \pm \sqrt{\kappa^{2} T^{2}+4 \omega^{2}}}{2}
$$

where the non-linear term is controlled by an $\omega$ parameter. The sources are supposed to be ruled by a perfect fluid energy-momentum tensor $T^{\mu \nu}=p g^{\mu \nu}+(p+\rho) U^{\mu} U^{\nu}$. The choose of the sign in the field equation (4) is related to the preservation of the JT limit [6]; to guarantee the existence of this limit one must follow the sign of the energy-momentum trace $T$ during the evolution of the system. The scalar curvature for the metric (2) in given by $R=\frac{2 \ddot{a}}{a}[6]$. Using these expressions in the field equation (4) (together with the energy-momentum tensor conservation law) we obtain the following system of equations

$$
\frac{2 \ddot{a}}{a}=\frac{-\kappa(\rho-p) \pm \sqrt{\kappa^{2}(\rho-p)^{2}+4 \omega^{2}}}{2}, \quad \dot{a}(\rho+p)+a \dot{\rho}=0
$$

where the gravitational source ( a mixture of inflatons and matter) is supposed to obey [6] the van der Waals (vdW) equation of state

$$
p=\frac{b \rho}{1-\alpha \rho},
$$

where $b$ and $\alpha$ are constant parameters; the use of the vdW equation in cosmological models was proposed in [7] and used in 2D models in [6].

The differential equations system (5) can be solved numerically. As a starting point, the following (normalized) initial conditions were used $a(0)=1, \dot{a}(0)=1$ e $\rho(0)=1$; these conditions simulate a 2D young Universe, at the beginning of an strongly accelerated (inflationary) period [6]. As we mentioned before the choice of sign in equations (4) depends on the behavior of $T$. What we verify is that the sign of $T$ separate naturally the time interval in two periods (being negative in the first period and positive in the second).

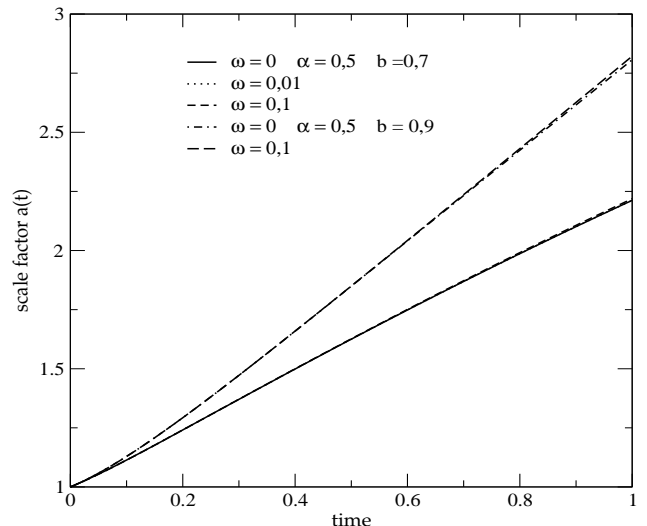

(a)

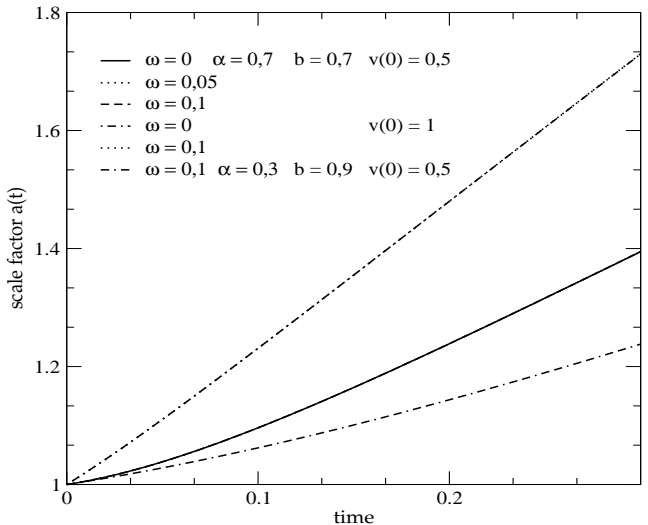

(b)

Fig. 1 - Evolution in time for scale factor a(t), for different values of the parameters, first period.

In figure (11) we present the evolution in time of the scale factor $a(t)$ for different values of the parameters. The first result observed is that the expansion becomes faster when either 
the parameters $b$ or $\alpha$ are increased, which is in accord with the behavior verified in [6]. More importantly, the results show that the presence of the non-linear term (using different values of the control parameter $\omega$ ) does not affect the expansion (notice the superimposition of curves in figures (a) and (b); the term in $1 / R$ is in fact buried by the presence of the energy-momentum trace term. This is in accord with the idea that the non-linear term play the role of the 2D dark energy constituent, becoming important only at later times (second period).

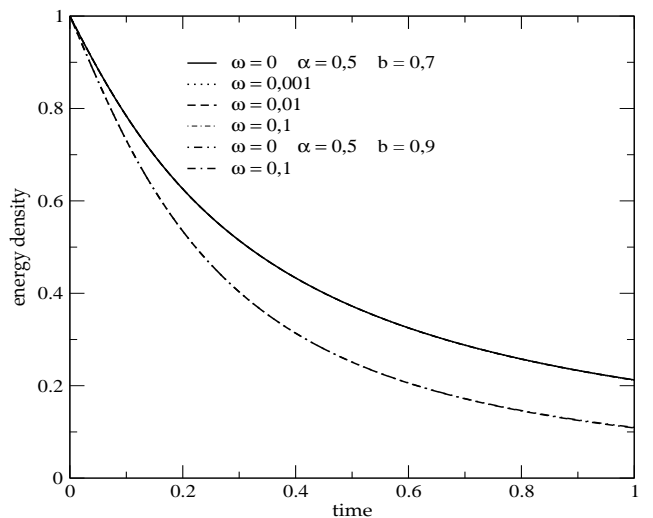

Fig. 2 - Evolution in time for the vdW energy density, taking different values of the parameters, first period.

In figure (2) we display the evolution of the energy density $\rho(t)$ of the vdW constituent. As expected, considering the behavior of the scale factor, the function $\rho(t)$ is in a ever decreasing regime; increasing values of $b$ (and $\alpha$, not displayed here) promote a faster fall on the values of $\rho(t)$. Changes in $\omega$ don't modify the behavior of the energy density for the early $2 \mathrm{D}$ universe.

The acceleration $\ddot{a}(t)$, as shown in figure (3) (a) and (b), is also not modified by the presence of the non-linear term in $R$. Increasing values of $\alpha$ turn the fall of the curve more drastic (together with an increasing value of the initial acceleration $\ddot{a}(0)$ ), approaching the turning point to a decelerated regime where matter starts to predominate (as the vdW equation of states approaches a barotropic equation as time increases).

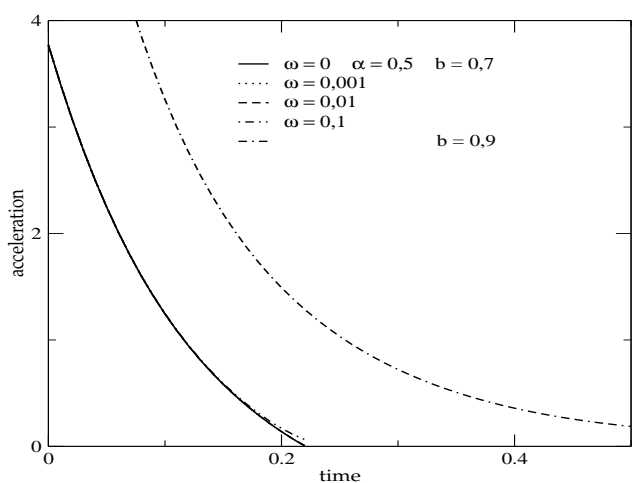

(a)

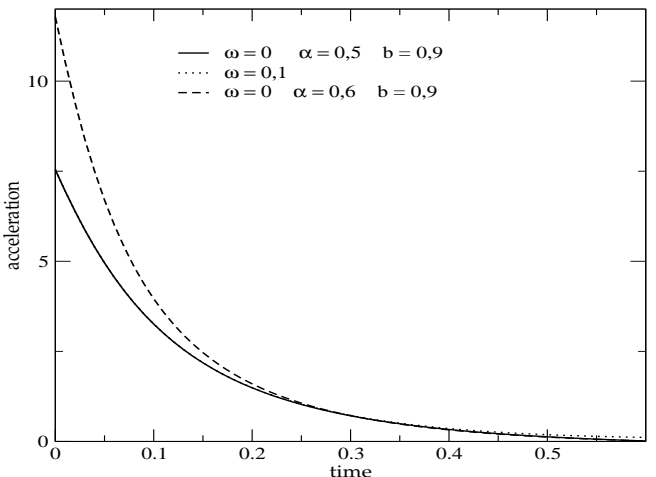

(b)

Fig. 3 - Evolution in time for acceleration $\ddot{a}(t)$ taking different values of the parameters, first period. 
In figures (4) and (5) we display the behavior of the scale factor $a(t)$, the vdW energy density and the acceleration $\ddot{a}(t)$ for the second period (when $T>0$ ). The curves show an ever expanding $2 \mathrm{D}$ universe (in some cases a big crunch situation emerges but only for later times $(t \approx 10)$ ), with increasing values of $\alpha$ and $b$ furnishing a faster expansion, as it was in the case of the first period. An expected behavior for the vdW energy density is also obtained in this case (not displayed), that is, a ever drecreasing function in an expanding $2 \mathrm{D}$ universe. The results related to the acceleration, in figure (5) show an strong dependence on fine tuning of the values of parameters $\alpha, b$ and $\omega$ and more importantly it is evident that the solely presence of the non-linear term does not guarantee a final accelerated era for the $2 \mathrm{D}$ universe.
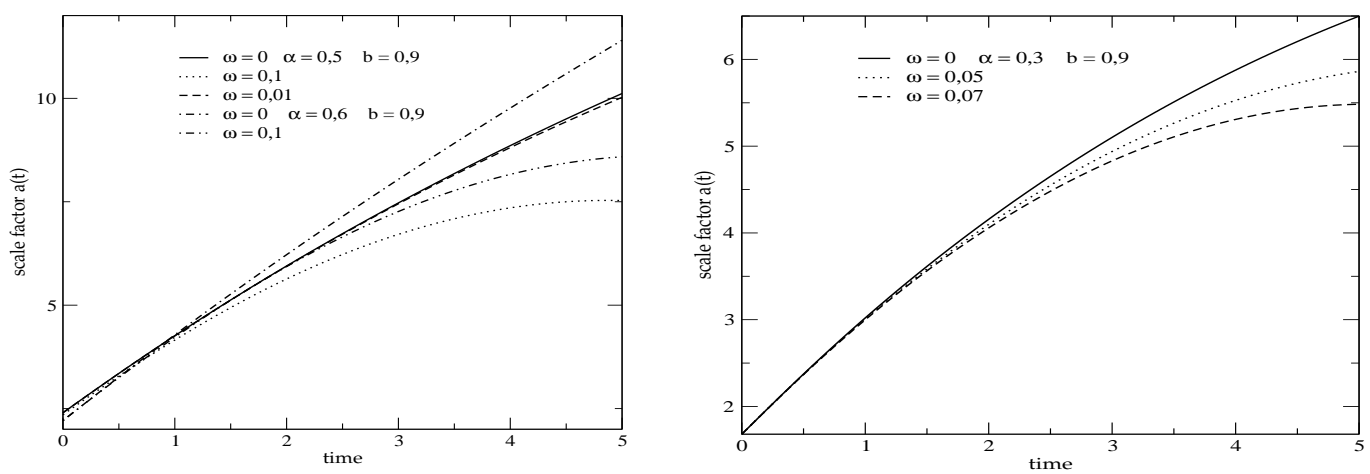

Fig. 4 - Evolution in time for scale factor a(t) taking different values of the parameters, second period.

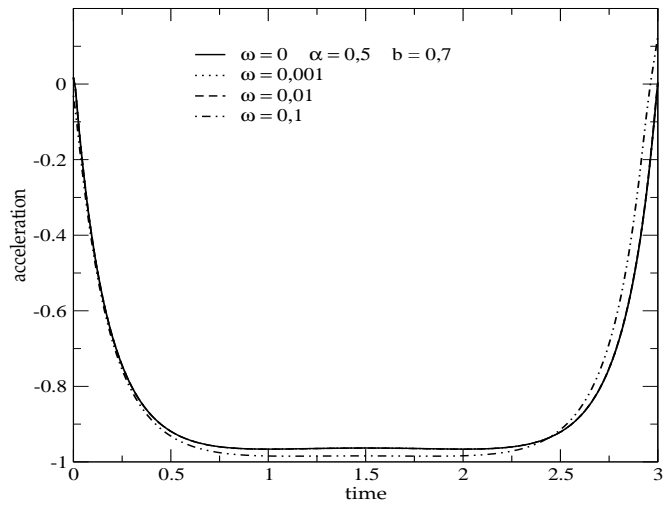

(a)

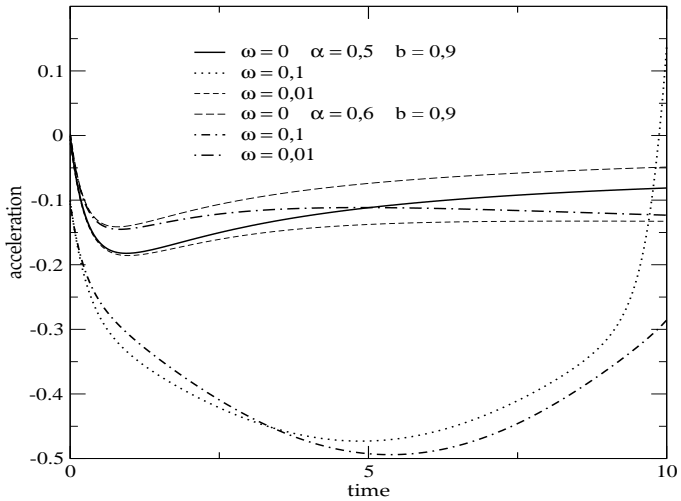

(b)

Fig. 5 - Evolution in time for acceleration $\ddot{a}(t)$ taking different values of the parameters, second period.

Next we investigate the model when the JT limit is not taken into account; that means, once one of the roots of equation (4) is chosen the sign is mantained for all times (independently of the sign of the trace $T$ ). The main point here is that in this case we get similar transitions to the ones found in [6], a three-eras regime, and more interestingly is that the non-linear term is effectively promoting a final accelerated regime, as it is explained bellow.

In figure (6)-(a) we display the behavior of the scale factor $a(t)$. The results show an ever expanding 2D universe, with increasing values of $\alpha$ and $b$ furnishing a faster expansion as in the previous case. Figure (6)-(b) shows (again) an expected behavior for the vdW energy density; 
increasing values of parameters $\alpha$ and $b$ furnish a more drastic fall of the curve with the passing of time. Only for later times the presence of the non-linear term in $R$ promotes a sensitive increasing in the expansion rate of this $2 \mathrm{D}$ universe.

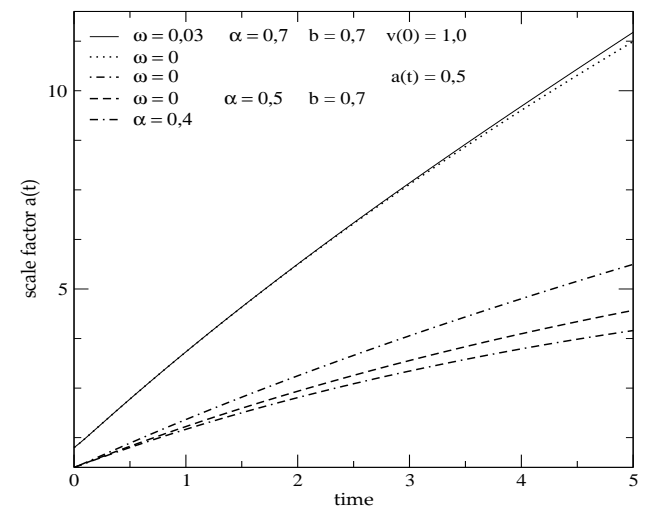

(a)

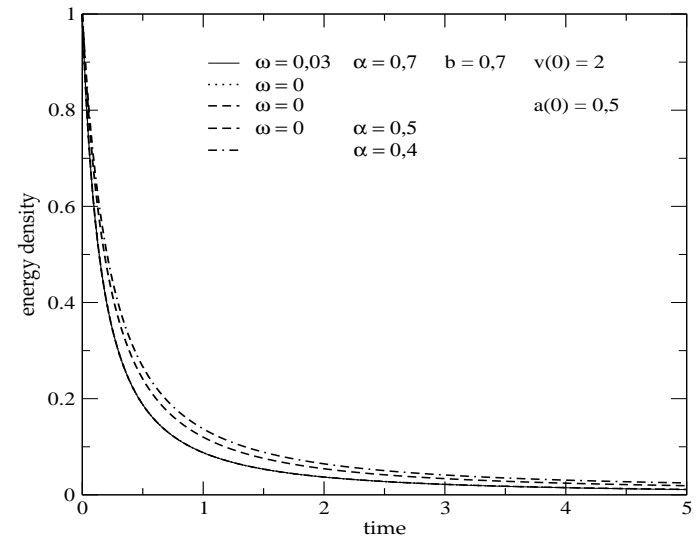

(b)

Fig. 6 - Evolution in time for scale factor $a(t)$ and energy density $\rho$, for different values of the parameters, without JT limit.

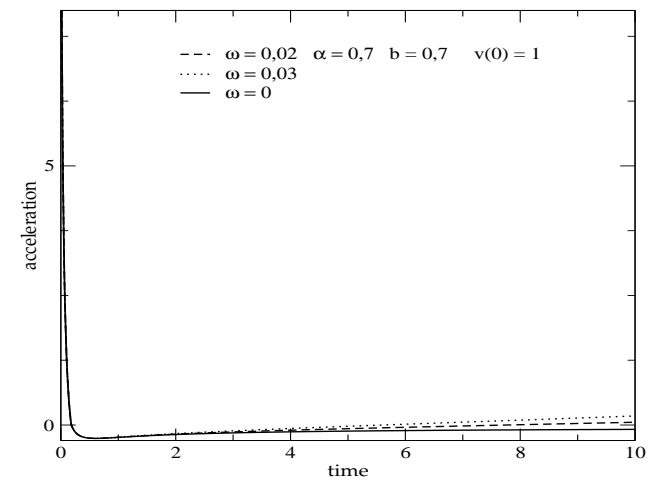

(a)

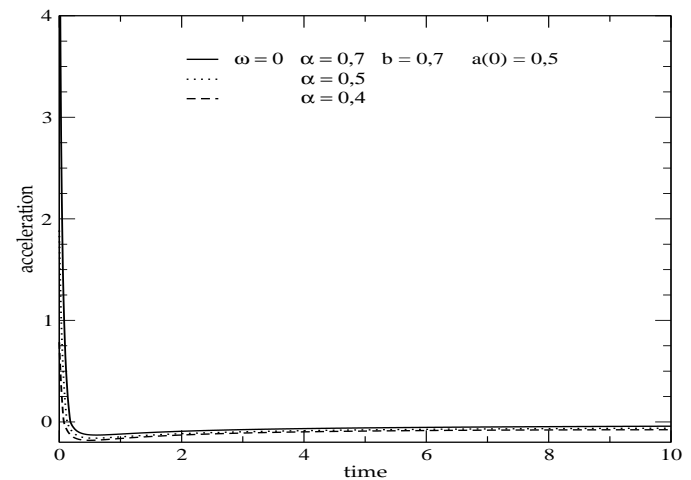

(b)

Fig. 7 - Evolution in time for acceleration $\ddot{a}(t)$, for different values of the parameters, without JT limit.

The behavior of the acceleration is the most interesting result, as we display in figure (77). As the values of time increase the term in $1 / R$ becomes more proeminent and boosts a transition between a decelerated era (dominated by matter, as far as the vdW equation of state is becoming a barotropic one as the system evolves) and a accelerated period. This indicates that the nonlinear term is playing a role similar to the cosmological constant (see [6]) but in this case the responsible for that final accelerated era has a geometrical origin. The effect happens in a more drastic way as parameter $\omega$ increases. Besides, increasing values of parameters $\mathrm{b}$ and $\alpha$ promote an earlier transition to the final accelerated regime. Although the case with the JT limit is strongly suceptible to changes in the initial conditions the case without the JT limit also shows situations where the association of the non-linear term with the dark energy is not possible. For instance, 
for the conditons $a(0)=1 v(0)=.9$ and $\rho(0)=.9$ a ciclic $2 \mathrm{D}$ universe emerges with a similarly ciclic behavior of the acceleration $\ddot{a}(t)$. Finally we would like to articulate some comments on the results obtained in this work. First, we should mention that the scalar theory (in the form of the JT model) is a consistent theory of gravity only in 2D and hence the properties of the cosmological scenarios that emerge can be exclusively associated to $2 \mathrm{D}$ space-times. In the $4 \mathrm{D}$ case several experimental tests show that a scalar formulation $(R \sim T)$ doesn't work even in considerable simple situations [3]. One alternative is the Brans-Dicke formulation that includes an additional field in the form of the dilaton (there is a $2 \mathrm{D}$ counterpart to Brans-Dicke that is known as the CGHS model [1]; the cosmological results are of difficult interpretation in that case [6]). Second, the substitution of the cosmological constant with the non-linear term in $R$ is interesting because it avoids the problem, that is exclusive of $2 \mathrm{D}$ gravity, of modelling the dark energy contribution as a source with a negative energy term. Third, a consistent quantum analysis of $2 \mathrm{D}$ gravitational models exists [1] and therefore quantum effects can be included in a full quantum scenario or in a semi-classical cosmological formulation [1]; these subjects are under investigation by the authors.

Conclusions . - In this work we have investigated the possibility of a term of the form $1 / R$ (as a correction to the Jackiw-Teitelboim equations) be responsible for an final accelerated regime in an old $2 \mathrm{D}$ universe. This term is substituing the usual idea of modelling the dark energy as a usual source (like an scalar field or a cosmogical constant) by giving it a geometrical origin. The model is rich enough to include several regimes, furnishing in special cases a three period behavior: a first strongly accelerated period corresponding to a young $2 \mathrm{D}$ universe, a second period dominated by matter/radiation and a final transition when the non-linear term starts to show its effects and promotes the desired final accelerated regime. On the other hand the model is very sensible to changes in initial conditions and the solutions include regimes where the $2 \mathrm{D}$ cosmogical interpretations are obscure.

\section{REFERENCES}

[1] D. Grumiller et al., Phys. Rept. 369, 327(2002) and references therein; J.D. Brown, Lower dimensional gravity (World Scientific, Singapore, 1993) and references therein.

[2] C. Teitelboim, in Quantum Theory of Gravity, edited by S. Christensen (Hilger, Bristol, 1984)p. 327; R. Jackiw, ibid p. 403.

[3] R. B. Mann and S. F. Ross, Phys. Rev. D 47, 3312 (1993); M. Cadoni and S. Mignemi, Gen. Rel. Grav. 34, 2101 (2002); G. M. Kremer and F. P. Devecchi Phys. Rev. D 65, 12 (2002).

[4] G. L. Murphy, Phys. Rev. D 8, 4231 (1973).

[5] D. N. Vollick, Phys. Rev. D 68, 063510 (2003); D. S. Alves and G. M. Kremer, JCAP 0410,0109 (2004).

[6] M.H. Christmann, F.P. Devecchi, G.M. Kremer, and C.M. Zanetti, Europhys. Lett. 67, 728 (2004).

[7] S. Capozziello, S. Martino, M. Falanga, Phys. Lett. A 299,494 (2002). 